\title{
Experimental Demonstration of a Bottle Microresonator
}

\author{
Ganapathy Senthil Murugan, James S. Wilkinson and Michalis N. Zervas \\ Optoelectronics Research Centre, University of Southampton, Southampton SO17 1BJ, United Kingdom. \\ Corresponding author E-mail: smg@orc.soton.ac.uk
}

\begin{abstract}
We demonstrate a very simple technique to fabricate robust microbottle resonators. Spheroidal WGMs and bottle modes were excited preferentially using a tapered fiber coupled at specific locations along the bottle, and characteristic resonance spectra obtained.

(C)2008 Optical Society of America

OCIS codes: $220.4000,140.4780,230.5750,060.4005,140.3948$
\end{abstract}

\section{Introduction}

The whispering gallery mode (WGM) bottle microresonator was proposed recently and has been studied theoretically [1-3]. Double-neck bottle-shaped microresonators have a number of features that distinguish them from equatorial WGM resonators such as microspheres and microtoroids. Bottle resonators sustain WGMs that exhibit two well-separated spatial regions with enhanced field strength [2]. The free spectral range (FSR) of such resonators is predicted to be about one order of magnitude smaller than that of microsphere resonators of equal diameter [2]. This is because bottle microresonators can also be considered as generalized Fabry-Perot cavities where "skew" rays are totally reflected at the two turning points close to the bottle necks. This results in a much longer optical path-length and, therefore, decreased FSR. The reduced FSR is particularly useful when tuning of a high-Q WGM is required to match atomic transition lines in experiments such as CQED [2].

We have fabricated fiber WGM bottle microresonators from short sections of optical fiber by a simple and versatile technique using only a standard fiber fusion splicer as found in every optical fiber lab. In this paper, we detail the new fabrication technique and present the first experimental observation of the intensity maxima at the turning points of the bottle resonator and the respective spectral characteristics.

\section{Bottle microresonator fabrication}

Fiber splicing is a thermo-mechanical process in which the cleaved ends of two optical fibers are pushed towards each other while they are heated to a temperature at which they soften and fuse together. The heating is usually performed in one of two ways, either by resistive coil (filament) heating or by arc discharge. We have exploited these splicer actions on a piece of continuous telecommunications fiber in order to soften a small region while simultaneously compressing it. These combined splicer actions produce a pronounced bulge along the fiber as shown in Fig. 1(a). The heating method used in the present study was arc discharge with an arc duration of about one second. We used multiple short arcs in order to controllably soften the glass. This procedure results in a robust, double-neck bottle fiber microresonator with neck-to-neck distance $L_{\mathrm{b}}$, bottle diameter $D_{\mathrm{b}}$ and stem diameter $D_{\mathrm{s}}$. The detailed "bottle" shape is also an important parameter in defining the spectral characteristics and optical properties of the resonator. The shape is defined by the softening temperature profile and the applied compression.
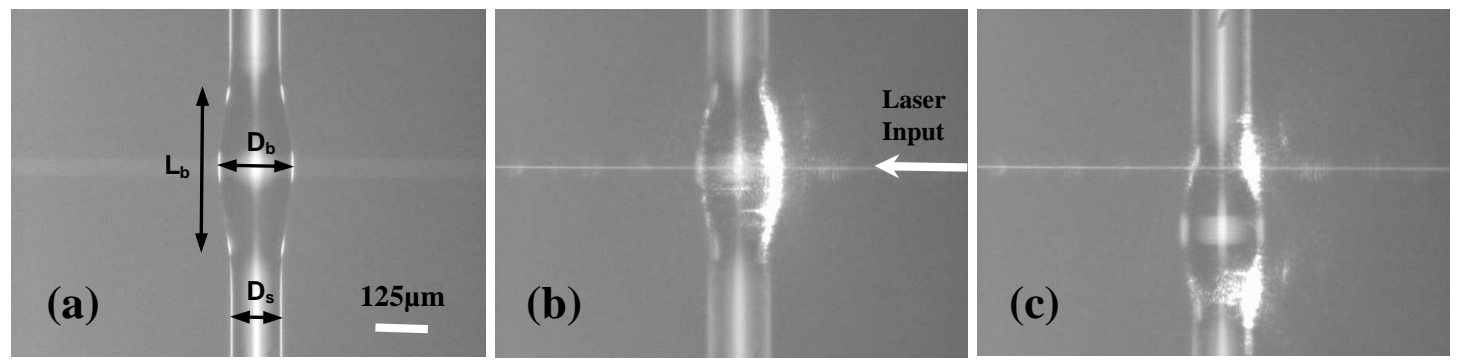

Fig. 1. (a) Optical micrograph of the bottle resonator coupled to a tapered optical fiber (focus on the bottle), (b) Image of the bottle resonator when the light was coupled through the tapered fiber at the centre (focus on the tapered fiber) and (c) Image of the bottle

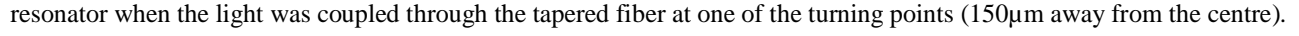

\section{Bottle microresonator characterization}

A tapered fiber with a waist diameter of $2 \mu \mathrm{m}$ was used to couple light into and out of the bottle resonator. The micro-bottle dimensions were $D_{\mathrm{s}}=125 \mu \mathrm{m}, D_{\mathrm{b}}=185 \mu \mathrm{m}$ and $L_{\mathrm{b}}=400 \mu \mathrm{m}$. Fig. 1(b) and (c) show images of the resonator when excited using a tapered fiber coupled at the center and at one of the turning points of the bottle resonator, respectively. The difference in the way the WGMs are excited in these two different positions is readily observed. The image in Fig. 1(c) clearly shows the excitation of a bottle mode with the characteristic 


\section{JTuD87.pdf}

intensity maxima on both sides of the bottle in the vicinity of the turning points, in close qualitative agreement with theoretical predictions [1,2]. However the image in Fig. 1(b) shows the excitation of different type of mode, resembling the conventional WGMs of a highly deformed sphere (spheroid) with non-degenerate mode splitting due to strong ellipticity. The latter although expected to be supported by bottle microresonators have not been studied extensively so far [1,2]. Depending on the relative field overlaps, the different types of modes are expected to be predominantly excited at different positions of the tapered fiber.

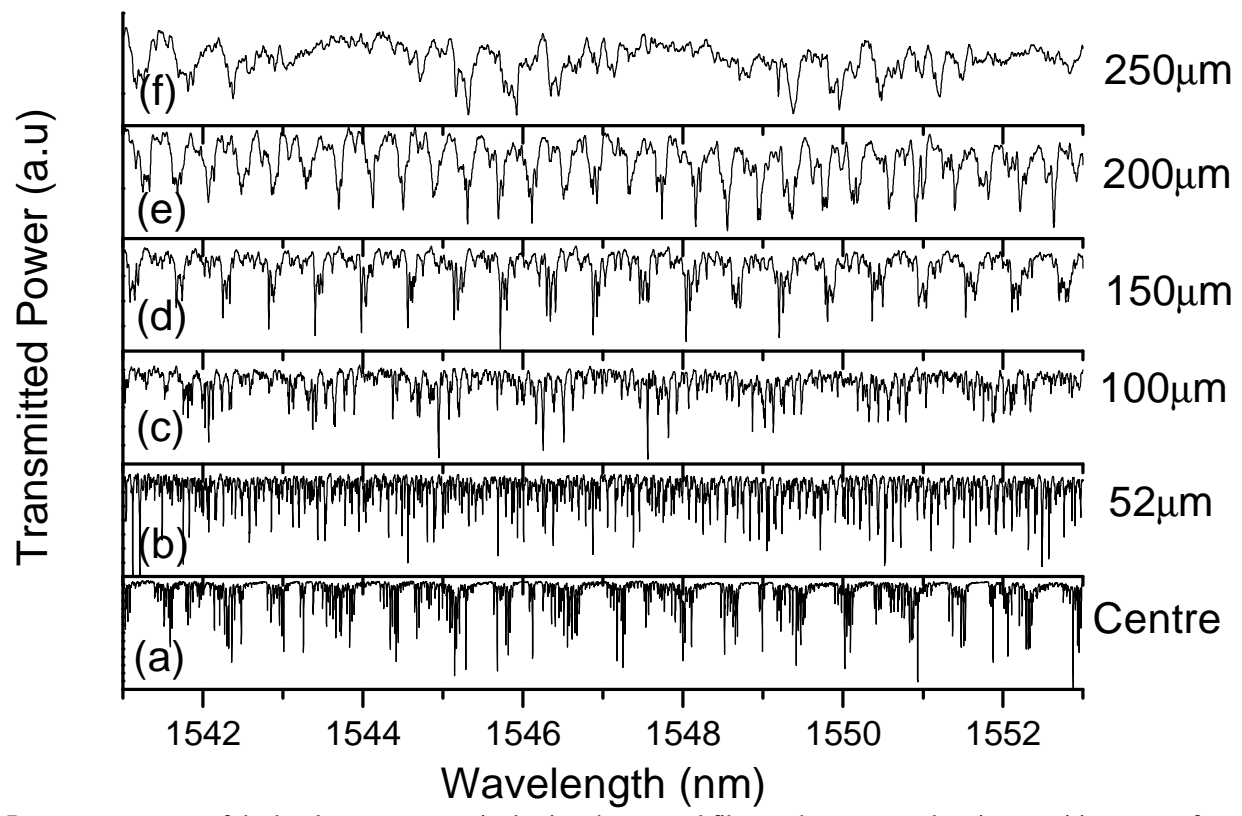

Fig. 2. Resonance spectra of the bottle resonator excited using the tapered fiber at the centre and various positions away from the centre.

Fig. 2 (a)-(f) show the resonance spectra of the bottle microresonator when excited at various positions along the bottle. The gradual transformation of the spectra from very dense deformed-sphere modes (excited at and around the centre) to the typical bottle modes is clearly seen as the turning points (about 150-200 $\mu \mathrm{m}$ away from the centre) are approached. Beyond these points only the normal fiber WGMs are excited and the FSR of $4.5 \mathrm{~nm}$ observed in this case, Fig. 2(f), is in agreement with the WGMs expected in the original fiber of cladding diameter $125 \mu \mathrm{m}$. The FSR observed for the bottle modes in Fig. 2(e) was about $0.4 \mathrm{~nm}$ near a wavelength of $1550 \mathrm{~nm}$, which is about one seventh of the FSR expected for a microsphere with the same diameter $(\approx 185 \mu \mathrm{m})$ as the bottle. This FSR is also equivalent to that of a standard FP cavity of about 5 times the length. This increased optical path-length is due to the long helical path followed by the WGM, travelling back and forth between the two turning points close to the bottle necks. This can also be considered equivalent to an effective group velocity slowing down by a factor of 5 , associated with the bottle modes.

\section{Conclusions}

In summary, we have experimentally demonstrated for the first time, the existence of bottle modes with spatially-separated intensity maxima at the turning points, a characteristic feature of the bottle microresonators. The reduced FSR observed is expected to be beneficial in CQED experiments since it requires much lower tuning effort (e.g. mechanical strain) to fully cover the FSR. In addition, compared to microspheres the WGMs supported by bottle microresonators occupy a much larger fraction of the resonator volume. This is expected to have a significant effect on the efficiency of optically pumped WGM bottle microresonator lasers. Work is underway to demonstrate these features.

\section{Acknowledgements}

The authors thank Dr. Yongmin Jung for providing the tapered fiber coupler and Dr. Yuh Tat Cho for providing the fusion splicer. This work was funded by the UK EPSRC under grant GR/S96500/01.

\section{References}

1. M. Sumetsky, "Whispering-gallery-bottle microcavities: the three-dimensional etalon," Opt. Lett. 29, 8-10 (2004).

2. Y. Louyer, D. Meshede, and A. Rauschenbeutel, "Tunable whispering-gallery-mode resonators for cavity quantum electrodynamics," Phys. Rev. A 72, 031801(R) (2005).

3. M. L. Gorodetsky and A. E. Fomin, "Geometrical theory of whispering-gallery modes," IEEE J. Sel. Top. Quant. Elec. 12, 33-39 (2006). 